\title{
Surgical management and prognostic factors for primary intracranial myxoma: a single-institute experience with a systematic review
}

\author{
*Jian-Cong Weng, MD, ${ }^{1}$ Lai-Rong Song, MD,' Da Li, MD, ${ }^{1}$ Liang Wang, MD, PhD, ${ }^{1}$ \\ Zhen Wu, MD, PhD, ${ }^{1}$ Jun-Mei Wang, MD, ${ }^{2}$ Gui-Lin Li, MD, ${ }^{2}$ Wang Jia, MD, PhD, ${ }^{1}$ \\ Li-Wei Zhang, MD, PhD, ${ }^{1}$ and Jun-Ting Zhang, MD' \\ 'Department of Neurosurgery, Beijing Tiantan Hospital, Capital Medical University; and 'Department of Neuropathology, Beijing \\ Neurosurgical Institute, Beijing, People's Republic of China
}

\begin{abstract}
OBJECTIVE Primary intracranial myxomas (PICMs) are extremely rare neoplasms, and their management and prognostic factors remain ambiguous. The authors aimed to elaborate the radiological features, evaluate the risk factors for progression-free survival (PFS), and propose a treatment protocol based on pooled data from cases treated at their institute and those found in the literature.
\end{abstract}

METHODS Clinical data from all cases of PICMs treated at the authors' institute and those cases reported in the English-language literature between 1987 and December 2017 were reviewed. The authors searched the Ovid MEDLINE, Embase, PubMed, and Cochrane databases using the keywords "myxoma" and "central nervous system," "intracranial," "cerebral," "skull base," "skull," or "brain." Previously published data were processed and used according to Preferred Reporting Items for Systematic Reviews and Meta-Analyses (PRISMA) guidelines. Risk factors in the pooled cohort were evaluated.

RESULTS Cases from the authors' institute included 21 males and 9 females, with a mean age of $35.7 \pm 1.7$ years. Gross-total resection (GTR) and non-GTR were achieved in $6(20.0 \%)$ and $24(80.0 \%)$ patients, respectively. After a mean follow-up of $86.7 \pm 14.1$ months, recurrence occurred in $6(24 \%)$ patients, for a median PFS time of 85.2 months (range 36.0-136.0 months) and no deaths. In the literature between 1987 and 2017, 35 cases of PICM were identified in 14 males and 21 females with a mean age of $31.7 \pm 3.2$ years. GTR and non-GTR were achieved in $23(65.7 \%)$ and 9 $(25.7 \%)$ cases, respectively. After a mean follow-up of $25.8 \pm 6.9$ months (range 1.0-156.0 months), recurrence occurred in $4(14.3 \%)$ patients, for a median PFS time of 11.0 months (range 3.0-36.0 months) and no deaths. Actuarial PFS rates at 1,5 , and 10 years were $93.0 \%, 80.6 \%$, and $67.9 \%$, respectively. A multivariate model demonstrated that GTR (HR $0.058,95 \% \mathrm{Cl} 0.005-0.680, \mathrm{p}=0.023$ ) was the only factor that favored PFS.

CONCLUSIONS PICMs are rare neoplasms with a slightly higher occurrence in males. GTR was the only favorable factor for PFS. Based on statistical results, GTR alone, if tolerable, is advocated as the optimal treatment for PICM. Nevertheless, conservative excision may be preferred to avoid damage to vital structures. PICMs have a tendency to recur within a few years of the initial surgery if resection is incomplete; therefore, close postoperative follow-up is mandatory. Future studies with larger cohorts are necessary to verify the study findings.

Systematic review registration no.: CRD42018091517 (www.crd.york.ac.uk/prospero/)

https://thejns.org/doi/abs/10.3171/2018.6.JNS181132

KEYWORDS central nervous system; primary intracranial myxoma; skull base; treatment strategy; oncology

ABBREVIATIONS BTH = Beijing Tiantan Hospital; $\mathrm{CN}=$ cranial nerve; $\mathrm{CNS}=$ central nervous system; $\mathrm{EMA}=$ epithelial membrane antigen; EMPT = estimated mean PFS time; GFAP = glial fibrillary acidic protein; GKS = Gamma Knife surgery; GTR = gross-total resection; IMRT = intensity-modulated RT; PFS = progression-free survival; PICM = primary intracranial myxoma; $\mathrm{RT}=$ radiotherapy.

SUBMITTED April 24, 2018. ACCEPTED June 20, 2018.

INCLUDE WHEN CITING Published online November 30, 2018; DOI: 10.3171/2018.6.JNS181132.

* J.C.W., L.R.S., and D.L. contributed equally to this work. 
$\mathrm{M}$ YXOMAS are rare, histologically benign tumors that arise from mesenchymal tissues throughout the body. They are most frequently found in the atrium of the heart and jaw bone and occur less frequently in the cranial base of the brain. ${ }^{16,33,35,37,38}$ From an etiological perspective, intracranial myxomas have either a primary or secondary origin. Initially, the majority of primary myxomas are found at the skull base, whereas secondary intracranial myxomas are mainly caused by metastatic tumor emboli from cardiac myxomas. ${ }^{40}$ Given the paucity of information regarding these tumors in the published literature, their management and prognostic factors remain ambiguous. Therefore, we retrospectively reviewed data on all patients who had undergone surgical treatment at our institute for pathologically confirmed primary intracranial myxomas (PICMs), and we performed an extensive literature review. We aimed to elaborate the radiological features of PICMs, evaluate the risk factors for progression-free survival (PFS), and propose a treatment protocol based on the pooled data from our cases and those in the literature.

\section{Methods \\ Patient Population}

The Beijing Tiantan Hospital (BTH) Research Ethics Committee approved this study. All patients with pathologically confirmed PICMs treated at BTH in the period from 1983 to 2017 were eligible for inclusion into the present study. Patients with metastatic myxomas were excluded. The clinical charts, surgical records, and radiographs for included patients were all reviewed. The following information was recorded: age, sex, main preoperative complaint, symptom duration, degree of tumor excision, tumor characteristics (size, volume, location, MRI and CT features), and any follow-up data.

Tumor size was evaluated and expressed as the lesion equivalent diameter $(a b c)^{1 / 3}$ (i.e., cube root of the product of the values of these 3 diameters), where $a, b$, and c were mutually orthogonal and represented the maximal diameters (width, thickness, height) measured on axial, sagittal, and coronal MRI scans. Evaluations (cubature formula: volume $=[\mathrm{a} \times \mathrm{b} \times \mathrm{c}] / 2)$ of both pre- and postoperative tumor volumes based on contrast MRI scans were performed to determine extent of resection, which was recorded as gross-total resection (GTR; without residue) or non-GTR (residue was noted on postoperative contrast MRI scans before discharge). Tumor consistencies were semi-quantitatively defined by our 2 senior surgeons (Chung-Cheng Wang and Jun-Ting Zhang) and classified into the following subtypes: 1) soft/crisp: could be aspirated by suction; 2) tenacious: could not be aspirated but could be cut with scissors; or 3) hard: could not be cut with scissors and demonstrated calcification on CT scans or contained calcified components.

Clinical and radiological follow-up were performed every 6 months within the first 2 years and then yearly thereafter through outpatient follow-up or telephone interviews.

\section{Pathological Examination}

Fresh paraffin-embedded tumor tissue was cut into
5- $\mu \mathrm{m}$ slices and stained with $\mathrm{H} \& \mathrm{E}$. Immunohistochemical staining for cytokeratin, epithelial membrane antigen (EMA), glial fibrillary acidic protein (GFAP), S100, vimentin, and desmin was used for the differential diagnosis of the samples. Two independent neuropathologists (J.M.W. and G.L.L.) reviewed the microscopic pathologies of the PICM samples. Diagnoses were established based on the typical histopathological characteristics of the PICMs.

\section{Literature Search Strategy}

The search protocol, including the research questions and inclusion and exclusion criteria, was developed a priori according to the Preferred Reporting Items for Systematic Reviews and Meta-Analyses (PRISMA) guidelines. This study was registered with the PROSPERO database (http://www.crd.york.ac.uk/prospero/), and its registration no. is CRD42018091517. We performed a search of the Ovid MEDLINE, Embase, PubMed, and Cochrane Library databases from 1987 to December 2017 (Fig. 1). The keywords used for the searches were "myxoma" and "central nervous system," "intracranial," "cerebral," "skull base," "skull," or "brain."

\section{Case Eligibility Criteria}

Inclusion criteria for both BTH and literature cases were as follows: 1) pathological diagnosis of myxoma; 2) intracranial and/or skull lesion location; and 3) detailed information on treatment, neurological outcome, status of recurrence, or death. Exclusion criteria consisted of the following: 1) lesion location other than intracranial or skull, 2) unavailable or incomplete clinical data, 3) basic research rather than clinical report without detailed clinical data, 4) undefined pathological diagnosis, or 5) duplicated report.

The following workups were performed to confirm a primary central nervous system (CNS) myxoma in our series: 1) all patients (not limited to the present cohort) routinely underwent preoperative echocardiography examination at our institute to confirm the cardiac disease-free status and to preclude the possibility of an atrial myxoma; 2) preoperative chest radiography and physical examination were performed to preclude potential myxoma of other parts of the body outside the CNS; 3) past medical history was rechecked; 4) preoperative MRI and CT scans were reviewed to differentiate primary myxomas (usually solitary and located in the extraaxial skull base) from metastatic ones (usually multiple and located in the parenchyma in the supratentorial region with hemorrhages, hemosiderin staining, and/or multiple aneurysms); 5) after confirming the pathological diagnosis of a myxoma after surgery, a systemic check-up, including chest and abdominal CT and abdominal ultrasound examination, was performed before discharge; and, most importantly, 6) whether a myxoma was present outside the CNS was reconfirmed during the postoperative follow-up evaluation.

\section{Study Eligibility}

Two reviewers (J.C.W. and L.R.S.) independently and in duplicate performed title and abstract screening of the 


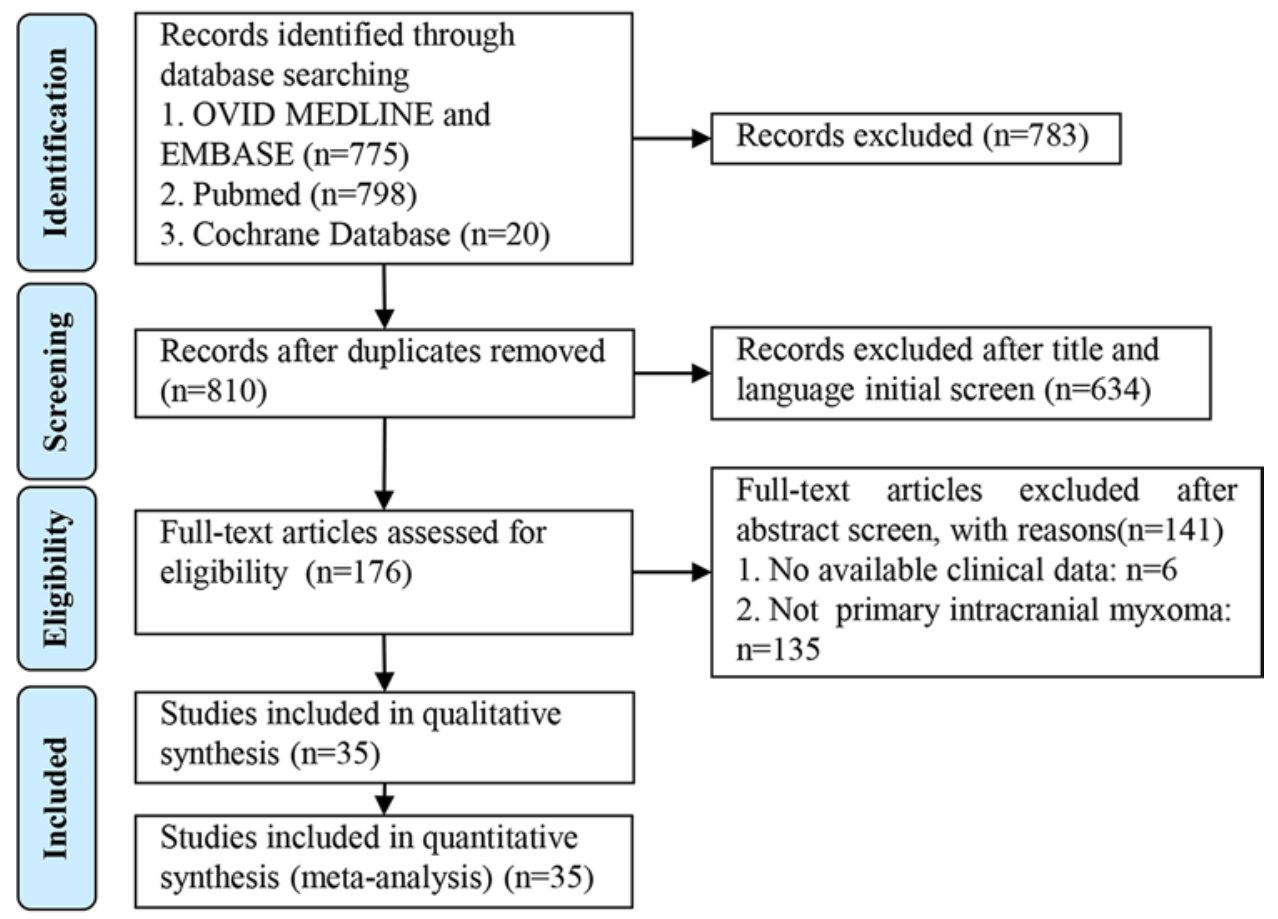

FIG. 1. PRISMA chart showing the inclusion and exclusion process for the analysis. Figure is available in color online only.

studies in the search query results. The full texts of eligible studies were obtained, and the reviewers resolved all disagreements through discussion or consultation with a third reviewer (D.L.) when needed.

\section{Statistical Analysis}

The primary outcome for patients with PICM was PFS. The pertinent risk factors were evaluated with univariate and multivariate Cox regression analysis of pooled cases (total 53 cases). Because only 17 of the 35 cases from the literature review provided information regarding tumor size, this factor was excluded from Cox regression analysis to avoid possible selection bias. The risk factors for overall survival could not be calculated because there were no deaths during the follow-up period. Secondary outcomes were PFS in the subgroups with significant risk factors, and the pertinent estimated mean PFS time (EMPT) was calculated using the Kaplan-Meier method (log-rank test). Because the percentage of censored cases was greater than 50\%, we used the EMPT to illustrate PFS. Analyses were performed using SPSS Statistical Package software (version 20.0, IBM Corp.) with significance set at $\mathrm{p}<0.05$.

\section{Results}

\section{Beijing Tiantan Hospital Cases \\ Patient Demographics}

The cases from BTH included 21 males and 9 females, with a mean age of 35.7 years (range 16-57 years; Table 1). The mean duration of symptoms was 28.0 months (range 0-156.0 months), and the most common preoperative symptoms were visual field defects (11 cases), followed by facial numbness ( 9 cases), diplopia ( 8 cases), headache
( 8 cases), and hearing loss/tinnitus ( 3 cases). Cranial neuropathies were observed in $29(96.7 \%)$ patients, and cranial nerve (CN) II palsy (11 cases) was the most common type, followed by CN V (9 cases), CN XII (8 cases), CN III (7 cases), and CN VI (7 cases) palsy. Two patients had undergone surgical treatment before being admitted to our institute (Supplementary Table 1).

\section{Preoperative Radiological Evaluation and Diagnosis}

Twenty-six patients had both preoperative CT and MRI scans, 2 patients had CT scans only, and the remaining 2 patients had MRI scans only. None of the patients had surgery without preoperative imaging. Two patients did not undergo CT examination, and 3 patients underwent $\mathrm{CT}$ at another institute, but these Digital Imaging and Communications in Medicine (DICOM) formatted data were unavailable to us. During the early period of our series, before 1986, 2 patients did not undergo MRI because it was unavailable. For another 2 patients, MRI was performed at another hospital, and images were unavailable for radiological evaluation. Overall, CT and MRI scans were available for radiological evaluation for 25 and 26 patients, respectively. On CT scans, osteolytic changes were demonstrated in 12 cases (48.0\%), and calcification was demonstrated in 13 cases $(52.0 \%)$. The most common manifestations on MRI were hypointensity on T1weighted images (21 cases [80.8\%]) and hyperintensity on T2-weighted images (21 cases [80.8\%]). Eleven of 16 lesions were heterogeneously enhanced on contrast images (MRI studies unavailable for 10 lesions; Fig. 2). Overall, lesions were derived from the skull base and mainly arose from the synchondrosis joint where cartilage is converted into bone after adolescence. Lesions predominantly arose 
TABLE 1. Clinical data in our series and in literature cases

\begin{tabular}{|c|c|c|c|c|}
\hline Variable & BTH Cases & Literature Cases & Total & $p$ Value \\
\hline No. of patients & 30 & 35 & 65 & \\
\hline Sex, no. (\%) & & & & $0.016^{*} \dagger$ \\
\hline Male & $21(70.0)$ & $14(40.0)$ & $35(53.8)$ & \\
\hline Female & $9(30.0)$ & $21(60.0)$ & $30(46.2)$ & \\
\hline Age in yrs & & & & $0.235 \ddagger$ \\
\hline Median & 35.0 & 33.0 & 35.0 & \\
\hline Mean \pm SD & $35.7 \pm 1.7$ & $31.7 \pm 3.2$ & $33.5 \pm 1.9$ & \\
\hline Main complaint, no. (\%) & & & & $0.002^{*} \dagger$ \\
\hline Visual field defect & $11(36.7)$ & $4(11.4)$ & $15(23.1)$ & \\
\hline Hearing loss/tinnitus & $3(10.0)$ & $13(37.1)$ & $16(24.6)$ & \\
\hline Diplopia & $8(26.7)$ & $4(11.4)$ & $12(18.5)$ & \\
\hline Headache & $8(26.7)$ & $14(40.0)$ & $22(33.8)$ & \\
\hline Facial numbness & $9(30.0)$ & $1(2.9)$ & $10(15.4)$ & \\
\hline Duration of symptoms in mos & & & & $0.086 \ddagger$ \\
\hline Median (range) & $12.0(0-156.0)$ & $4.0(0-96.0) \S$ & $12.0(0-156.0) \S$ & \\
\hline Mean \pm SD & $28.0 \pm 6.8$ & $12.0 \pm 4.9 \S$ & $22.6 \pm 5.2 \S$ & \\
\hline Synchondrosis of origin, no. (\%) & & & & $0.001^{*} \dagger$ \\
\hline Petroclival & $3(10.0)$ & $2(5.7)$ & $5(7.7)$ & \\
\hline Sphenopetrosal & $7(23.3)$ & $5(14.3)$ & $12(18.5)$ & \\
\hline Sphenoethmoidal & $3(10.0)$ & $8(22.9)$ & $11(16.9)$ & \\
\hline Sphenooccipital & $7(23.3)$ & 0 & $7(10.8)$ & \\
\hline Petrooccipital & $7(23.3)$ & $11(31.4)$ & $18(27.7)$ & \\
\hline Calvarium bone & 0 & $4(11.4)$ & $4(6.2)$ & \\
\hline Multiple origins & $3(10.0)$ & $1(2.9)$ & $4(6.2)$ & \\
\hline Indeterminate origin & 0 & $4(11.4)$ & $4(6.2)$ & \\
\hline Resection, no. (\%) & & & & $<0.001^{*} \dagger$ \\
\hline GTR & $6(20.0)$ & $23(65.7)$ & $29(44.6)$ & \\
\hline Non-GTR & $24(80.0)$ & $9(25.7)$ & $33(50.8)$ & \\
\hline NA & 0 & $3(8.6)$ & $3(4.6)$ & \\
\hline FU duration in mos & & & & $<0.001^{*} \ddagger$ \\
\hline Range & $4.0-258.0 \rrbracket$ & $1.0-156.0^{* *}$ & 1.0-258.0†† & \\
\hline Mean \pm SD & $86.7 \pm 14.1 \rrbracket$ & $25.8 \pm 6.9^{* *}$ & $63.6 \pm 11.8 \dagger \dagger$ & \\
\hline Recurrence/progression, no. (\%) & $6(24.0) \Uparrow$ & $4(14.3)^{\star *}$ & $10(18.9) \dagger \dagger$ & $0.582 \dagger$ \\
\hline \multicolumn{5}{|l|}{ KPS score } \\
\hline On admission & $80.6 \pm 1.3$ & NA & NA & \\
\hline At discharge & $70.3 \pm 3.9$ & NA & NA & \\
\hline At FU & $78.1 \pm 4.6$ & NA & NA & \\
\hline \multicolumn{5}{|l|}{ Neurological outcome, no. (\%) } \\
\hline Improved & $2(6.7)$ & NA & NA & \\
\hline Stable & $15(50.0)$ & NA & NA & \\
\hline Worsened & $13(43.3)$ & NA & NA & \\
\hline Death during FU & 0 & 0 & 0 & \\
\hline
\end{tabular}

FU = follow-up; KPS = Karnofsky Performance Scale; NA = not available.

${ }^{*} p<0.05$.

$\dagger$ Chi-square test.

$\ddagger$ Independent sample t-test.

$\S$ Fifteen patients did not have available data.

I Three patients were lost to follow-up, and 2 patients died of postoperative complications.

** Seven patients did not have available follow-up data.

†† Twelve patients did not have available follow-up data. 

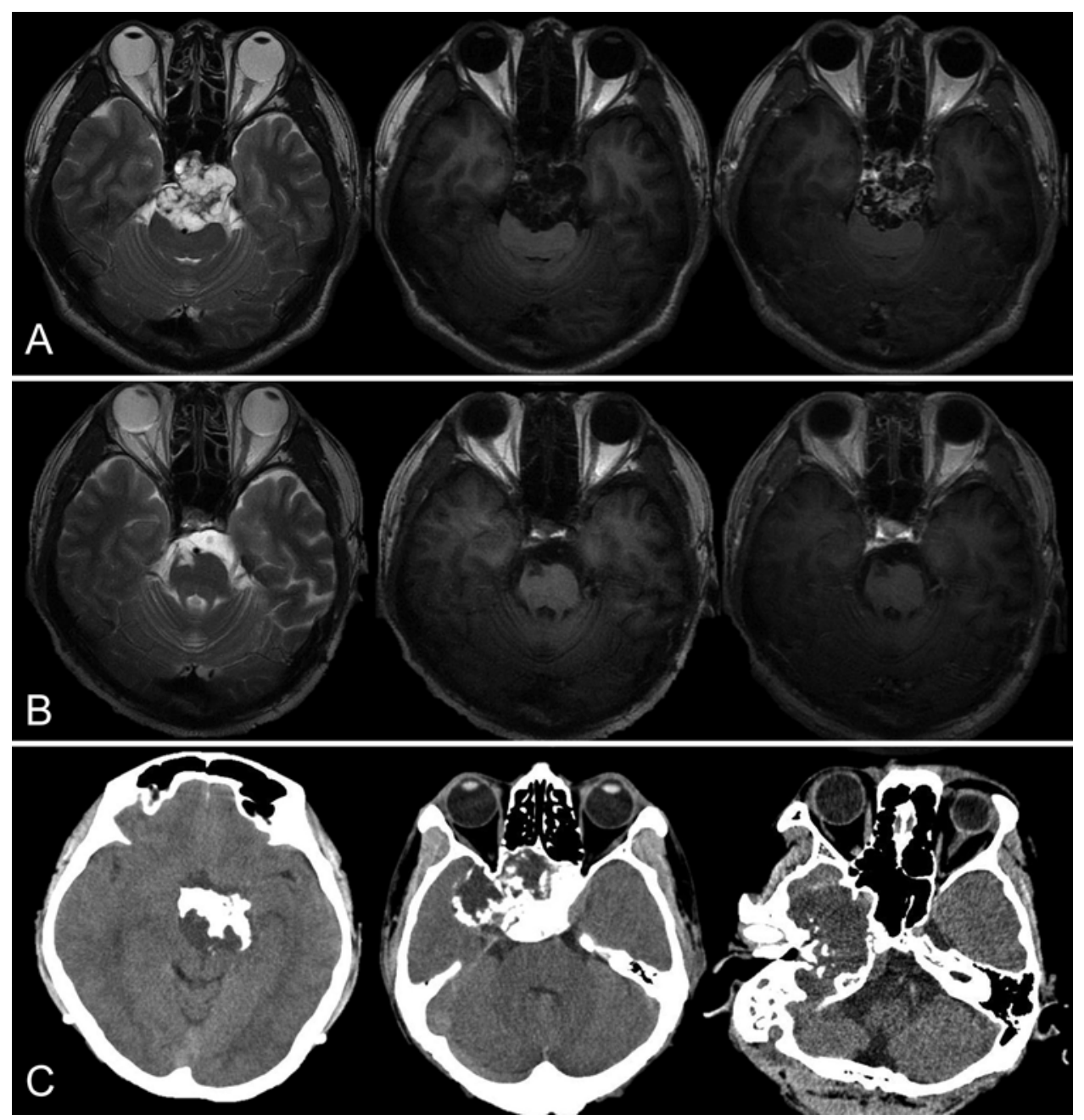

FIG. 2. A case of PICM in a 27-year-old male whose main complaint was intermittent headaches and diplopia that had been occurring for 12 months. Preoperative MR images (A) showed a lesion located in the clival area expanding into the ethmoid sinus and mixed isointense-hypointense signal and mixed isointense-hyperintense signal on T1- and T2-weighted imaging, respectively, and heterogeneous enhancement on contrast MRI. After GTR, he received no additional treatment. Contrast MR images (B) showed complete resection without recurrence after 162 months. PICMs can manifest as bony growths and osteolysis on preoperative CT scans (C).

from the sphenopetrosal synchondrosis (7 cases), sphenooccipital synchondrosis (7 cases), and petrooccipital synchondrosis (7 cases; Tables 1 and 2). Preoperative radiological diagnosis included chordomas (6 cases), followed by schwannomas (5 cases), craniopharyngiomas (4 cases), and chondrosarcomas (3 cases).

\section{Surgical Findings}

Several surgical approaches were used for the PICMs, including the frontotemporal approach (13 cases [43.3\%]), retrosigmoid approach (6 cases [20.0\%]), presigmoid approach (4 cases [13.3\%]), frontoorbital approach with or without the transzygomatic approach (3 cases [10.0\%]), far lateral approach (2 cases [6.7\%]), and anterior transpetrosal approach (2 cases [6.7\%]). Under a microscope, the lesions varied in color from grayish-white (14 cases) to grayish-red (10 cases) and grayish-yellow (6 cases). The surgical plane was distinguishable in 22 lesions and was clear in only 8 lesions. The tumor consistency was soft and subjected to suction in 13 cases, tenacious in 7 cases, and mixed soft/tenacious in 10 cases. The lesions manifested with bone destruction of the skull base (18 cases) and bone hyperplasia (12 cases). Intraoperative blood loss volumes ranged from 100 to $3700 \mathrm{ml}$, with a mean of 900.0 $\pm 233.8 \mathrm{ml}$. Based on postoperative MRI scans, GTR and non-GTR were achieved in $6(20.0 \%)$ and $24(80.0 \%)$ patients, respectively. Two patients died due to postoperative hematomas and respiratory failure. Other complications included a cranial infection (1 case) and a massive cerebral infarction (1 case; Supplementary Table 1).

\section{Follow-Up Outcomes}

Three patients were lost to follow-up after discharge. Thus, only 25 patients were available for survival analysis. Postoperative adjuvant radiotherapy (RT) was administered to 8 patients; 7 patients received intensity-modulated 
TABLE 2. Tumor characteristics in pooled cases

\begin{tabular}{|c|c|}
\hline Characteristic & No. $(\%)$ \\
\hline \multicolumn{2}{|l|}{ Size in $\mathrm{cm}$} \\
\hline Mean \pm SD & $38.7 \pm 2.5$ \\
\hline Range & $11.5-88.5$ \\
\hline \multicolumn{2}{|l|}{ Lesion vol in $\mathrm{cm}^{3}$} \\
\hline Mean \pm SD & $56.5 \pm 12.9$ \\
\hline Range & $1.0-346.0$ \\
\hline \multicolumn{2}{|l|}{ MRI feature } \\
\hline T1 \& T2 signals & $44(100)$ \\
\hline Hypo T1 \& hyper T2 & $30(68.2)$ \\
\hline Hypo T1 \& mixed iso/hyper T2 & $2(4.5)$ \\
\hline Mixed iso/hypo T1 \& mixed iso/hyper T2 & $4(9.1)$ \\
\hline $\begin{array}{l}\text { Mixed iso/hypo T1 \& mixed iso/hypo/ } \\
\text { hyper T2 }\end{array}$ & $1(2.3)$ \\
\hline Mixed iso/hypo T1 \& hyper T2 & $1(2.3)$ \\
\hline Iso T1 \& hyper T2 & $2(4.5)$ \\
\hline Iso T1 \& mixed iso/hyper T2 & $1(2.3)$ \\
\hline T1 NA \& hyper T2 & $3(6.8)$ \\
\hline \multicolumn{2}{|l|}{ Edema } \\
\hline Peritumor edema & $7(15.9)$ \\
\hline No peritumor edema & $37(84.1)$ \\
\hline Enhancement & $37(100)$ \\
\hline Heterogeneous & $19(51.4)$ \\
\hline Homogeneous & $10(27.0)$ \\
\hline Circular & $7(18.9)$ \\
\hline Nodular & $1(2.7)$ \\
\hline CT feature & $48(100)$ \\
\hline Bone destruction & $27(56.3)$ \\
\hline Calcification & $17(35.4)$ \\
\hline Neither & $4(8.3)$ \\
\hline Immunohistochemical results & $38(100)$ \\
\hline S100 positive/negative & $9(32.1) / 19(67.9)$ \\
\hline EMA positive/negative & $2(11.8) / 15(88.2)$ \\
\hline Vimentin positive/negative & $18(94.7) / 1(5.3)$ \\
\hline Cytokeratin positive/negative & $1(6.3) / 15(93.8)$ \\
\hline GFAP positive/negative & $1(10.0) / 9(90.0)$ \\
\hline Desmin positive/negative & $1(14.3) / 6(85.7)$ \\
\hline
\end{tabular}

hyper = hyperintensity; hypo = hypointensity; iso = isointensity .

RT (IMRT), and 1 patient received Gamma Knife surgery (GKS; Leksell Gamma Knife C). The total dose of fractionated local radiation received ranged from 30 to 57.6 Gy, with a mean and median dose of $43.9 \pm 3.9$ Gy and 50 Gy, respectively (single dose 1.8-2.0 Gy and 15-32 fractions). After a mean follow-up of $86.7 \pm 14.1$ months (range $4.0-258.0$ months), a total of 8 recurrences occurred in 6 patients (24\%; 5 patients had 1 recurrence, and 1 patient had 3 recurrences; Table 1), for a median PFS time of 85.2 months (range 36.0-136.0 months). Five patients underwent a second operation, one of whom underwent postoperative RT and another of whom underwent repeated surgery after each of three recurrences. Three patients who underwent repeat surgeries experienced postoperative complications, including intracranial hematoma (1 case), left-sided paralysis ( 1 case), hydrocephalus (1 case), and cerebrospinal fluid leakage (1 case). Overall, no patients died due to PICM.

\section{Data for Literature Cases}

For the period between 1987 and 2017, we identified 35 cases of PICM in 14 males and 21 females, with a mean and median age of $31.7 \pm 3.2$ years and 33.0 years, respectively (Tables 1 and 3). The most common preoperative symptom was headache (14 cases [40.0\%]), followed by hearing loss/tinnitus (13 cases [37.1\%]). The duration of symptoms ranged from 0 to 96.0 months (median 4.0 months). Lesions predominantly involved the petrooccipital synchondrosis (11 cases [31.4\%]) and sphenoethmoidal synchondrosis (8 cases [22.9\%]). GTR and non-GTR were achieved in $23(65.7 \%)$ and 9 (25.7\%) cases, respectively. After a mean follow-up of $25.8 \pm 6.9$ months (range 1.0156.0 months), recurrence occurred in 4 patients (14.3\%) for a median PFS time of 11.0 months (range 3.0-36.0 months) and no deaths.

\section{Histological Examination}

All 65 PICMs in our report exhibited similar histological features. Staining with H \& E showed that stellate cells were abundant in the myxoid matrix. Cytological atypia, increased mitotic activity, or necrosis was generally absent (Fig. 3). The immunohistochemistry results for the BTH group, in combination with those for the literature cases, were as follows: 19 of 28 PICMs were negative for S100 staining, 15 of 17 were negative for EMA staining, 18 of 19 were positive for vimentin staining, 15 of 16 were negative for cytokeratin staining, 9 of 10 were negative for GFAP staining, and 6 of 7 were negative for desmin staining (Table 2).

\section{Pooled Analysis: Factors Affecting PFS}

In the pooled cohort (53 cases), recurrence occurred in 10 patients (18.9\%). Results of univariate Cox regression analysis (including age, sex, skull base lesion location, no peritumor edema, GTR, bone destruction, RT, and S100 positivity) showed that compared with non-GTR (EMPT: 94.8 months), only GTR (EMPT: 153.2 months) had significantly prolonged PFS ( $p=0.019 ;$ Fig. 4). Actuarial PFS rates at 1,5 , and 10 years were $93.0 \%, 80.6 \%$, and $67.9 \%$, respectively. The estimated median survival time for PFS was 136.3 months. Whether postoperative RT can increase local control remains controversial; therefore, we added sex, age, RT, and GTR to the multivariate Cox regression analysis. GTR (HR 0.058, 95\% CI 0.005-0.680, $\mathrm{p}=0.023$ ) was the protective factor. Female sex (HR 1.425, 95\% CI $0.310-6.551, \mathrm{p}=0.649$ ), age (HR $0.947,95 \%$ CI $0.882-$ $1.017, \mathrm{p}=0.134)$, and RT (HR 0.118, 95\% CI 0.011-1.317, p $=0.083$ ) were nonsignificant factors (Fig. 5).

\section{Discussion}

Myxomas, which were first described by Virchow in 1863 , are benign tumors derived from mesenchymal origins $^{15,16}$ and usually occur in the heart. Primary intracra- 
TABLE 3. Literature review of PICM studies from 1987 to 2017

\begin{tabular}{|c|c|c|c|c|c|c|}
\hline Authors \& Year & Age/Sex (yrs) & Lesion Location & Tx & Recurr & FU (mos) & Status \\
\hline Nagatani et al., 1987 & $38 / \mathrm{M}$ & Posterior fossa & NA & No & 48 & NA \\
\hline Maiuri et al., 1988 & $64 / F$ & Frontotemporal & Non-GTR & Yes & 18 & Alive/wo \\
\hline Charabi et al., 1989 & $56 / \mathrm{M}$ & Temporal & NA & NA & NA & NA \\
\hline $\mathrm{Ng}$ et al., 1989 & $17 / \mathrm{F}$ & Parietooccipital & Non-GTR & No & 26 & Alive/wo \\
\hline Ito et al., 1990 & $11 / \mathrm{F}$ & Lat skull base & GTR & NA & NA & NA \\
\hline Klein et al., 1990 & $32 / F$ & Posterior fossa & NA & NA & NA & NA \\
\hline Shotton et al., 1992 & $35 / \mathrm{M}$ & Jugular foramen & GTR & No & 156 & Alive/wo \\
\hline Paulus et al., 1993 & $16 / F$ & Cavernous sinus & GTR & No & 12 & Alive/wo \\
\hline Knox et al., 1996 & $2 / \mathrm{F}$ & Lat skull base & GTR & NA & NA & NA \\
\hline Pitkäranta et al., 1997 & $16 / \mathrm{M}$ & Lat skull base & GTR & No & 12 & Alive/wo \\
\hline Graham et al., 1999 & $\mathrm{NA} / \mathrm{F}$ & Frontal & GTR & No & 60 & Alive/wo \\
\hline Srinivasan, 2000 & $14 / F$ & Temporal & GTR & No & 12 & Alive/wo \\
\hline Osterdock et al., 2001 & $17 / M$ & Temporal & Non-GTR & NA & NA & NA \\
\hline Windfuhr \& Schwerdtfeger, 2004 & $4 / F$ & Lat skull base & GTR & Yes & 120 & Alive/wo \\
\hline Sato et al., 2004 & $50 / F$ & Sphenoid sinus & GTR & No & 24 & Alive/wo \\
\hline Powers et al., 2006 & $39 / F$ & Falx cerebri & GTR & No & 1 & Alive/wo \\
\hline Medeiros et al., 2006 & $48 / F$ & Falx cerebri & GTR & No & 72 & Alive/wo \\
\hline Hsieh et al., 2006 & $32 / F$ & Temporal & GTR & No & 12 & Alive/wo \\
\hline Yin et al., 2007 & $27 / M$ & Temporal & Non-GTR & No & 6 & Alive/wo \\
\hline Moore et al., 2008 & $45 / \mathrm{M}$ & Sphenoid sinus & GTR & No & 12 & Alive/wo \\
\hline Menon et al., 2008 & $25 / M$ & Parietal & GTR & No & 3 & Alive/wo \\
\hline Mueller et al., 2010 & $12 / F$ & Posterior fossa & GTR & No & 11 & Alive/wo \\
\hline Sareen et al., 2010 & $11 / F$ & Temporal & GTR & No & 24 & Alive/wo \\
\hline Oruckaptan et al., 2010 & $34 / \mathrm{M}$ & Temporal & Non-GTR & Yes & 36 & Alive/wd \\
\hline Xiao et al., 2011 & $11 / \mathrm{M}$ & Sphenoid sinus & Non-GTR & Yes & 3 & Alive/wd \\
\hline Erdem et al., 2012 & $37 / \mathrm{F}$ & Clival & GTR & No & 1 & Alive/wo \\
\hline Guha-Thakurta et al., 2012 & $41 / \mathrm{M}$ & Temporal & Non-GTR & NA & NA & NA \\
\hline Alexandru et al., 2012 & $40 / \mathrm{M}$ & Posterior fossa & GTR & NA & NA & NA \\
\hline Klimo et al., 2013 & $13 / F$ & Lat skull base & GTR & No & 12 & Alive/wo \\
\hline Kawatra et al., 2013 & $12 / \mathrm{M}$ & Occipital & Non-GTR & No & 2 & Alive/wd \\
\hline Vij et al., 2013 & $45 / F$ & CPA & GTR & No & 6 & Alive/wo \\
\hline Ryu et al., 2015 & $50 / \mathrm{M}$ & Anterior fossa & Non-GTR & No & 3 & Alive/wd \\
\hline Sugita et al., 2016 & $55 / F$ & Falx cerebri & GTR & No & 3 & Alive/wo \\
\hline Bulduk et al., 2016 & $55 / F$ & Temporal & GTR & No & 2 & Alive/wo \\
\hline Clarke et al., 2017 & $72 / F$ & Sphenoid sinus & GTR & No & 24 & Alive/wo \\
\hline
\end{tabular}

$\mathrm{CPA}=$ cerebellopontine angle; recurr = recurrence; $\mathrm{Tx}=$ treatment; $\mathrm{wd}=$ with disease; wo = without disease.

nial myxomas are rare primary intracranial neoplasms. Limited data regarding PICMs are available in the literature, and most cases have been presented as isolated and anecdotal case reports. Here, we reported the largest series to date of 30 cases of PICMs located in the skull, and we systematically reviewed 35 cases of skull PICMs in the literature. Using our cases and those in prior studies, we verified adverse factors for PFS and found significant differences in PFS following various treatment modalities. Myxomas occur rarely as part of familial and inherited disorders such as Carney's complex or Marfan's syndrome: ${ }^{18}$ however, these associations were absent in the pooled cohort.

\section{Clinical Features and Radiological Features}

Patients in the BTH group were predominantly male, with a male/female ratio of 2.33:1, which was significantly different from the ratio in the literature. A slight male predilection was observed in the pooled cohort $(65$ cases), with a male/female ratio of 1.17:1, and the mean and median ages were $33.5 \pm 1.9$ and 35.0 years, respectively. Zhang et al ${ }^{40}$ insisted that cranial base myxomas appear to grow more rapidly in younger patients than in older patients. However, based on our pooled cases, there was no positive correlation between disease course and age (r (48) $=0.148, \mathrm{p}=0.306$ ); therefore, compared with older patients, younger patients did not have a faster tumor growth 


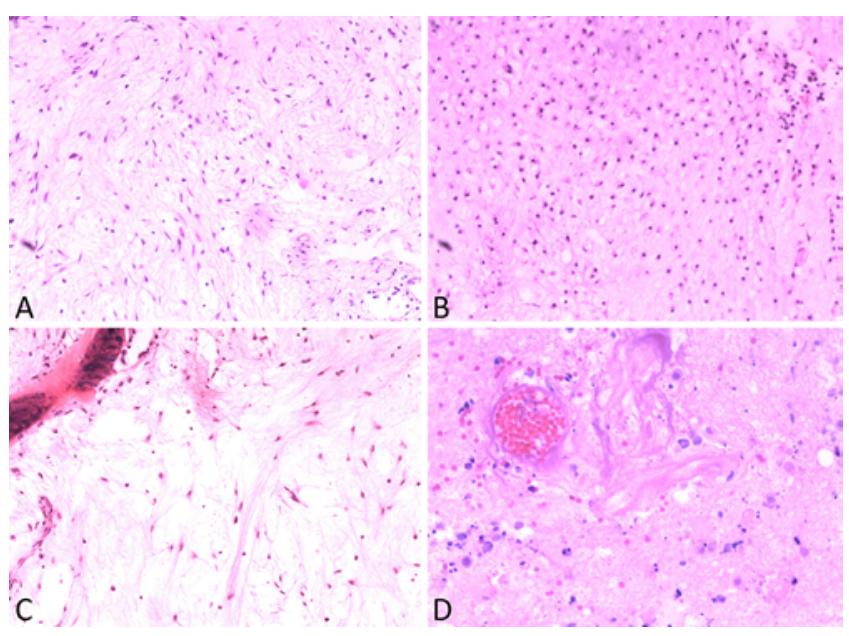

FIG. 3. Staining of PICMs with H \& E revealed stellate cells in an abundant myxoid matrix. Cytological atypia, increased mitotic activity, or necrosis is generally absent. Original magnification $\times 100$ (A and $B$ ) and $\times 400$ (C and D). Figure is available in color online only.

rate. PICMs mostly originate from the skull base; as a consequence, $\mathrm{CN}$ palsy is the chief complaint. When temporal bone lesions invade posteriorly, patients often present with conductive hearing loss and/or a mass in the middle ear. Patients with lesions involving the petrous apex often presented with vertigo and/or sensorineural hearing loss (16 cases [24.6\%]). When lesions invaded the optic canal, visual field defects often developed (15 cases [23.1\%]). Patients in the BTH group had a mean follow-up time of 86.7 \pm 14.1 months; in the literature, the mean follow-up time was $25.8 \pm 6.9$ months $(\mathrm{p}<0.001)$. For the BTH patients, the follow-up duration was longer to better present the prognostic effect and outcome.

Ryu et al. ${ }^{30}$ noted that radiological examination is a decisive tool for diagnosing myxomas. CT scans can reveal bone destruction and the clearness of tumor margins.
Myxomas can range from hypodense to isodense on CT scans and show variable enhancement patterns. Bone window imaging reveals the degree of bony destruction and the expansile pattern of the mass, and bony growth is rarely found with myxomas. ${ }^{28}$ However, in the pooled cases, $35.4 \%$ of the lesions manifested as bony growths. MRI had low signal intensity on T1-weighted images (32 cases [72.7\%]) and high signal intensity (36 cases [81.8\%]) and absence of edema (37 cases [84.1\%]) on T2-weighted images, with heterogeneous enhancement (19 cases [51.4\%]) detected frequently. PICMs can occur in any part of the cranial base but mainly occur at the bone joints, such as the sphenopetrosal (12 cases [18.5\%]), ${ }^{23}$ petrooccipital (18 cases [27.7\%]), sphenoethmoidal (11 cases [16.9\%]), and sphenooccipital (7 cases [10.8\%]) synchondroses. ${ }^{11,13}$ Preoperative diagnosis of schwannomas, craniopharyngiomas, and chondrosarcomas in the BTH group accounted for $16.7 \%, 13.3 \%$, and $10.0 \%$ of lesions, respectively. None of these lesions were preoperatively diagnosed as myxomas. Based on our study, preoperative radiological data are less reliable and insufficient for accurate diagnosis; additionally, pathological examination should be used to determine the diagnosis.

\section{Histological Characteristics of PICMs and Differential Diagnoses}

Immunohistochemistry is helpful in distinguishing PICMs. In the pooled cohort (38 cases), the main immunohistochemistry result was negative for S100, EMA, cytokeratin, desmin, and GFAP staining and positive for vimentin staining.

The differential diagnoses for PICMs include myxomatous meningioma, sarcomas with myxoid degeneration, and metastatic myxomas. ${ }^{18}$ EMA positivity is seen in patients who have meningiomas with myxoid components. Sarcoma stains positive for smooth muscle actin and desmin. True myxomas will stain positive for vimentin, suggestive of its mesenchymal origin, and not for any of the markers mentioned above. ${ }^{1,4,5,7,11,12,14,17,18,21,26,27,29,31,36}$
A

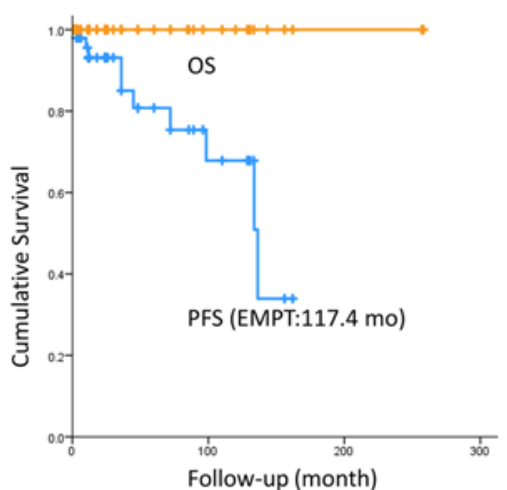

no. at risk

$\begin{array}{ll}\text { OS } & 53 \\ \text { PFS } & 53\end{array}$
B
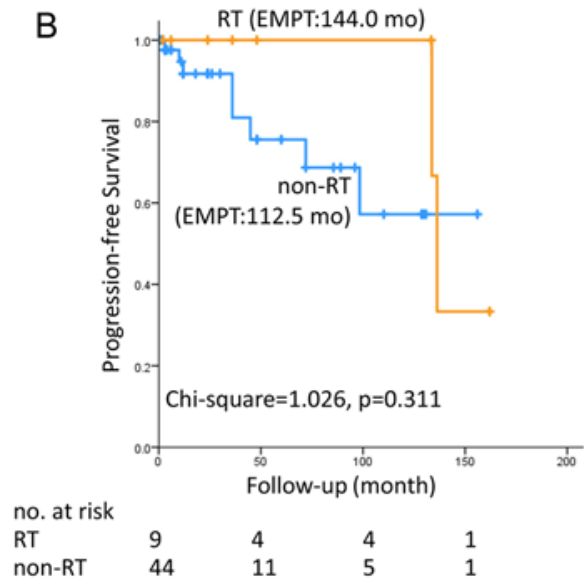

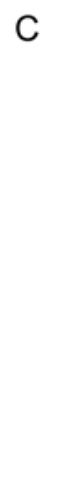

no. at risk non-GTR

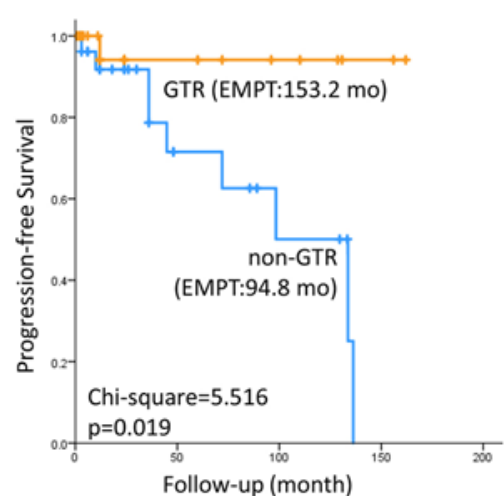

Follow-up (month)

$\begin{array}{llll}26 & 8 & 5 & 2 \\ 27 & 8 & 4 & \end{array}$

FIG. 4. Kaplan-Meier curve showing overall survival (OS) and PFS of the pooled cohort (A) and PFS of the pooled cohort divided into RT and non-RT groups (B) and GTR and non-GTR groups (C). According to the analysis, GTR was the only favorable factor for PFS. Figure is available in color online only. 


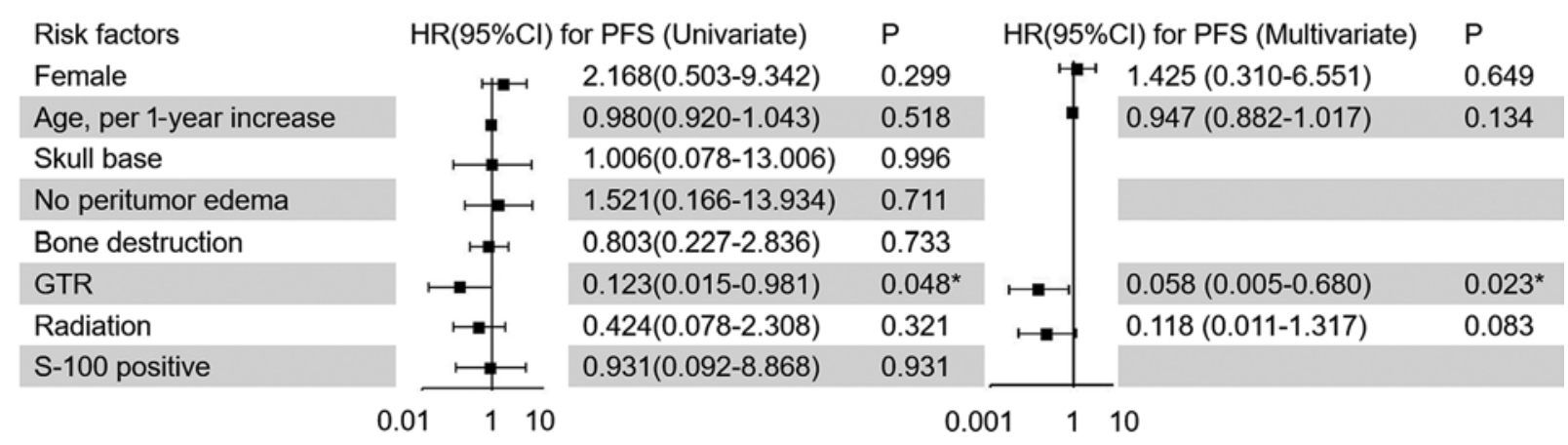

FIG. 5. Univariate and multivariate Cox regression analyses were used to evaluate the adverse factors for PFS. Black squares indicate the hazard ratio (HR) values, error bars represent the 95\% confidence intervals (Cls), and asterisks indicate $p<0.05$.

\section{Progression-Free Survival and Risk Factors}

The adverse factors affecting PFS are indefinite. Andrews et al. ${ }^{2}$ reported a recurrence rate of $6 \%$ with aggressive resection, which is a much lower rate than recurrences of $28 \%$ caused by inadequate surgery such as enucleation or curettage. With respect to RT, Guha-Thakurta et al. ${ }^{8}$ noted that it is used only to reduce the size of large tumors and that preoperative embolization can be used to decrease the vascularity during resection. Sato et al., ${ }^{32}$ Osterdock et al., ${ }^{24}$ and Srinivasan ${ }^{34}$ have reported that myxoid tumors are generally insensitive to RT and that postoperative RT has no therapeutic effect on myxomas of the bone in other areas; therefore, RT after surgery is unnecessary. However, Zhang et al. ${ }^{40}$ reported that among 8 cases receiving postoperative RT, 4 cases did not recur within the followup period. Three other cases receiving postoperative RT recurred 2, 3, and 6 years after discharge. One case had GKS; after 7 months, MRI demonstrated that the tumor had reduced in size. Zhang et al. ${ }^{40}$ believed that radiation treatment may have a positive effect on PICMs, but further study is required. In terms of immunohistochemistry, Pahl et $\mathrm{al} .{ }^{25}$ proposed that a propensity for tumor malignancy (myxosarcoma) and adverse prognoses can be expected when immunostaining of a neoplasm shows positive S100 protein expression.

Based on the literature, univariate Cox regression was used to evaluate risk factors for PFS from PICMs. The results indicated that age $(\mathrm{p}=0.518)$, postoperative $\mathrm{RT}(\mathrm{p}=$ $0.321)$, positive expression of $\mathrm{S} 100(\mathrm{p}=0.931)$, no peritumor edema $(p=0.711)$, tumor location $(p=0.996)$, and osteolysis $(p=0.733)$ did not worsen PFS. Postoperative RT remains controversial; thus, RT was included in the multivariate Cox regression analysis. The results confirmed that compared to GTR, non-GTR was the only independent risk factor for PFS. Patients with non-GTR suffered a 17.2fold increase in recurrence. There was no significant difference between RT (EMPT: 144.0 months) and non-RT (EMPT: 112.5 months; $p=0.311$ ). Two of the 7 cases in the BTH group that received IMRT had recurrences after 11.1 and 11.3 years. One case had GKS; after 13.5 years, MRI showed that the volume of the residual lesion had shrunk.

\section{Proposed Treatment Paradigm}

The treatment of choice for PICM is radical surgical removal because this lesion is generally insensitive to
RT. ${ }^{30}$ The majority of PICMs in the pooled cohort originated in the skull base (59 cases [90.8\%]). Because of their mucinous content, incomplete encapsulation, and proximity to important structures, only 6 cases $(20.0 \%)$ from our institute had GTR. Complete removal of lesions from the cranial base bone is difficult, especially when the lesions have widely and deeply infiltrated the skull base., ${ }^{9,15}$ Even if complete removal were feasible, the morbidity and mortality rates would be too high. Two patients were diagnosed with clival myxomas and died from postoperative intracranial hematoma and respiratory failure. Following gross-total removal of these tumors, reconstructing the cranial base can also be a challenge. Based on the results of a multivariate analysis, postoperative RT did not show any benefit. Mueller et al. ${ }^{20}$ reported an intracranial myxoma that had become a secondary neoplasia after RT. Ito et al. ${ }^{10}$ insisted that RT increases the chances of benign myxomas undergoing sarcomatous changes; therefore, RT is not advised when the lesion is not gross-totally removed, and close follow-up is necessary. ${ }^{3}$ Given the low chances of treatment with GTR and their insensitivity to RT, myxomas have a recurrence rate of $25 \%$ in periods varying from 3 months to 10 years, but they mostly recur within the first 2 years after initial treatment. Therefore, close follow-up is necessary for detecting PICM recurrence., ${ }^{9,19}$ The appearance of mucus on radiological imaging or newly developed clinical symptoms should be considered important indicators of PICM recurrence. ${ }^{40}$ Secondary surgical treatment is vital for recurrences.

\section{Study Limitations}

The main limitations of this study are its retrospective nature and limited sample size. First, it is a retrospective review of case reports on a rare disease over 30 years; thus, the rate of missing detailed data for survival analysis $(18.5 \%)$ is relatively high. The literature has a mean follow-up time of $25.8 \pm 6.9$ months, which cannot reflect a reliable prognostic result. Second, given the rarity of PICMs, there was a lack of randomized data, and potential bias may exist in the pooled statistical analysis data. Third, because the percentage of censored cases was greater than $50 \%$, the estimated median survival time could not be calculated. Consequently, the estimated mean survival time was used. We used EMPT to illustrate PFS. Theoretically, the median could provide a more appropriate estimate of 
typical survival time than the mean, and the estimated mean survival time grossly overestimated the PFS of patients with PICM and was inappropriate for extrapolating beyond the extent of the actual data.

Finally, our series had a long timespan from 1983 to 2017, and during the early period, MRI was unavailable. The subjective consistency measurement reflected a single-institute experience and was a limitation of the study; therefore, we defined it as a semi-quantitative consistency measurement according to intraoperative findings and the hardest part of the lesion. Given the intralesional heterogeneous consistency and the retrospective nature of the study, an objective quantitative consistency measurement was unavailable. In the BTH group, we indeed noted various consistencies of PICMs that needed to be clarified and reported within the neurosurgical community, but we did not further analyze the relation between consistency and PFS because of its subjectivity, which limited the repeatability, extrapolation, and accuracy of our results. Therefore, a prospective multicenter study with a large series of PICMs is recommended.

\section{Conclusions}

PICMs are rare neoplasms with a slightly higher occurrence in males. GTR was the only favorable factor for PFS. Based on statistical analysis, GTR alone, if tolerable, is advocated as the optimal treatment for PICM.,39 Nevertheless, conservative excision may be preferred to avoid damage to vital structures. ${ }^{22}$ PICMs have a tendency to recur within a few years of the initial surgery if resection is incomplete; therefore, postoperative follow-up is essential. Future studies with large cohorts are necessary to verify our findings.

\section{References}

1. Alexandru D, Satyadev R, So W: Neurothekeoma in the posterior fossa: case report and literature review. Perm J 16:63-64, 2012

2. Andrews T, Kountakis SE, Maillard AA: Myxomas of the head and neck. Am J Otolaryngol 21:184-189, 2000

3. Bulduk EB, Aslan A, Öcal Ö, Kaymaz AM: Neurothekeoma in the middle cranial fossa as a rare location: Case report and literature review. Neurochirurgie 62:336-338, 2016

4. Charabi S, Engel P, Bonding P: Myxoid tumours in the temporal bone. J Laryngol Otol 103:1206-1209, 1989

5. Clarke JV, Mandpe AH, Weber PB, Vogel H, Leng LZ: The unusual presentation of a myxoma within the sphenoid sinus: case report and review of the literature. World Neurosurg 103:951.e5-951.e12, 2017

6. Erdem Y, Koktekir E, Bayar MA, Yilmaz A, Caydere M: Characterization of an intracranial neurothekeoma: case report. Turk Neurosurg 22:109-112, 2012

7. Graham JF, Loo SY, Matoba A: Primary brain myxoma, an unusual tumor of meningeal origin: case report. Neurosurgery 45:166-170, 1999

8. Guha-Thakurta N, Deavers M, DeMonte F, Gidley PW: The natural history of primary temporal bone myxoma. Ann Diagn Pathol 16:280-283, 2012

9. Hsieh DL, Tseng HM, Young YH: Audiovestibular evolution in a patient undergoing surgical resection of a temporal bone myxoma. Eur Arch Otorhinolaryngol 263:614-617, 2006

10. Ito M, Tajima A, Nitta T, Sato K, Ishii S, Ragsdale B: Massive cranial ossifying myxoma in a child. Clin Neurol Neurosurg 92:271-275, 1990
11. Kawatra M, Bhandari V, Phatak S, Kulkarni D: Primary occipital myxoma: a rare case report. J Pediatr Neurosci 8:129-131, 2013

12. Klein MV, Schwaighofer BW, Sobel DF, Hesselink JR: Primary myxoma of the posterior fossa. Neuroradiology 32:250-251, 1990

13. Klimo P Jr, Jha T, Choudhri AF, Joyner R, Michael LM II: Fibromy xoma of the lateral skull base in a child: case report. J Neurol Surg Rep 74:105-110, 2013

14. Knox GW, Roth M, Saleh H, Stiles W: A unique temporal bone lesion resembling juvenile active ossifying myxoma. Am J Otol 17:297-300, 1996

15. Liu A, Wang CC: Primary myxomas of the skull base. J Clin Neurosci 3:29-33, 1996

16. Maiuri F, Corriero G, Galicchio B, Angrisani P, Bonavolontà G: Myxoma of the skull and orbit. Neurochirurgia (Stuttg) 31:136-138, 1988

17. Medeiros F, Scheithauer BW, Oliveira AM, Gregory RS: Angiomyxofibromatous tumor of the falx cerebri. Am J Surg Pathol 30:545-547, 2006

18. Menon RK, Goel A, Shah A, Goel N, Rajashekharan P: Primary intracranial myxoma of the parietal region. Illustrated case report. J Neurooncol 88:157-160, 2008

19. Moore BA, Wine T, Burkey BB, Amedee RG, Butcher RB II: Sphenoid sinus myxoma: case report and literature review. Ochsner J 8:166-171, 2008

20. Mueller OM, van de Nes JA, Wieland R, Schoch B, Sure U: Surgical treatment of primary intracranial myxoma in a child following radiotherapy: case report and review of the literature. Childs Nerv Syst 26:829-834, 2010

21. Nagatani M, Mori S, Takimoto N, Arita N, Ushio Y, Hayakawa T, et al: Primary myxoma in the pituitary fossa: case report. Neurosurgery 20:329-331, 1987

22. Ng SH, Tang LM, Wai YY, Tsai CC, Teh BC: Primary myxoma of the cranium: CT findings. J Comput Assist Tomogr 13:903-905, 1989

23. Oruckaptan HH, Sarac S, Gedikoglu G: Primary intracranial myxoma of the lateral skull base: a rare entity in clinical practice. Turk Neurosurg 20:86-89, 2010

24. Osterdock RJ, Greene S, Mascott CR, Amedee R, Crawford BE: Primary myxoma of the temporal bone in a 17-year-old boy: case report. Neurosurgery 48:945-948, 2001

25. Pahl S, Henn W, Binger T, Stein U, Remberger K: Malignant odontogenic myxoma of the maxilla: case with cytogenetic confirmation. J Laryngol Otol 114:533-535, 2000

26. Pal L, Bansal K, Behari S, Sagar BC, Gupta RK, Gupta RK, et al: Intracranial neurothekeoma-a rare parenchymal nerve sheath myxoma of the middle cranial fossa. Clin Neuropathol 21:47-51, 2002

27. Paulus W, Warmuth-Metz M, Sörensen N: Intracranial neurothekeoma (nerve-sheath myxoma). Case report. J Neurosurg 79:280-282, 1993

28. Pitkäranta A, Carpén O, Ramsay H: Fibromyxoma of the temporal bone. Otolaryngol Head Neck Surg 117:S201S203, 1997

29. Powers CJ, Pizzi CC, Cummings TJ, Friedman AH: Primary myxoma of the parafalcine meninges. Case report. J Neurosurg 104:440-443, 2006

30. Ryu JI, Cheong JH, Kim JM, Kim CH: A primary ossifying intracranial myxoma arising from the ethmoid sinus. J Korean Neurosurg Soc 58:281-285, 2015

31. Sareen D, Sethi A, Mrig S, Nigam S, Agarwal AK: Myxoma of the temporal bone: an uncommon neoplasm. Ear Nose Throat J 89:E18-E20, 2010

32. Sato H, Gyo K, Tomidokoro Y, Honda N: Myxoma of the sphenoidal sinus. Otolaryngol Head Neck Surg 130:378380, 2004

33. Shotton JC, Kuhwoede R, Fisch U: Mesenchymal tumors of the skull base with particular reference to surgical management and outcome. Skull Base Surg 2:112-117, 1992 
34. Srinivasan US: Fibromyxoma of the petrous apex. Pediatr Neurosurg 32:209-213, 2000

35. Sugita S, Obata M, Takebayashi S, Kikuchi T, Hasegawa T: Angiomyxomatous tumor of the falx cerebri. Pathol Int 66:50-51, 2016

36. Vij M, Jaiswal S, Agrawal V, Jaiswal A, Behari S: Nerve sheath myxoma (neurothekeoma) of cerebellopontine angle: case report of a rare tumor with brief review of literature. Turk Neurosurg 23:113-116, 2013

37. Windfuhr JP, Schwerdtfeger FP: Myxoma of the lateral skull base: clinical features and management. Laryngoscope 114:249-254, 2004

38. Xiao P, Liu D, Peng J, Gao Z, Dong Y, Wu H, et al: Aggressive angiomyxoma of the sphenoidal sinus. Pathology 43:79-81, 2011

39. Yin H, Cai BW, An HM, You C: Huge primary myxoma of skull base: a report of an uncommon case. Acta Neurochir (Wien) 149:713-717, 2007

40. Zhang L, Zhang M, Zhang J, Luo L, Xu Z, Li G, et al: Myxoma of the cranial base. Surg Neurol 68 (Suppl 2):S22-S28, 2007

\section{Disclosures}

The authors report no conflict of interest concerning the materials or methods used in this study or the findings specified in this paper.

\section{Author Contributions}

Conception and design: JT Zhang, Weng, Song, D Li, L Wang, Wu, JM Wang, LW Zhang. Acquisition of data: Weng, Song, D Li, L Wang, JM Wang, GL Li, Jia. Analysis and interpretation of data: all authors. Drafting the article: Weng, Song, D Li, Jia. Critically revising the article: all authors. Reviewed submitted version of manuscript: all authors. Approved the final version of the manuscript on behalf of all authors: JT Zhang, Statistical analysis: JT Zhang, Weng, Song, D Li, L Wang, JM Wang, LW Zhang. Administrative/technical/material support: JT Zhang, Weng, Song, D Li, L Wang, Wu, GL Li, Jia, LW Zhang. Study supervision: all authors.

\section{Supplemental Information}

\section{Online-Only Content}

Supplemental material is available with the online version of the article.

Supplementary Table 1. https://thejns.org/doi/suppl/10.3171/ 2018.6.JNS181132.

\section{Correspondence}

Jun-Ting Zhang: Beijing Tiantan Hospital, Capital Medical University, Beijing, People's Republic of China. zhangjunting2003@ aliyun.com. 